\title{
A Clinical Commentary on the Article "Biomaterials Advances in Patches for Congenital Heart Defect Repair"
}

\author{
Cardiac Bioengineering for Congenital Heart Disease: Time for Progress
}

\author{
Charles D. Fraser Jr.
}

Received: 1 June 2011 / Accepted: 10 June 2011 /Published online: 16 August 2011

(C) Springer Science+Business Media, LLC 2011

Congenital heart disease remains the most common birth defect affecting almost $1 \%$ of the population and if one considers congenitally bicuspid aortic valves, the number of affected individuals rises to as much as $4 \%$ by many estimates. The spectrum of congenital heart disease is broad. Patients may have very complex structural anomalies including an absence of a ventricle (single ventricle), lack of connection to a great artery (aortic or pulmonary atresia), anomalies of venous connections (systemic or pulmonary venous), valvar abnormalities (semilunar and/or atrioventricular), abnormal connections (transposition or malalignment), and vascular hypoplasia (aortic coarctation, hypoplasia, or interruption). Individuals affected by variations of these problems may present with acute circulatory compromise immediately at birth, later in infancy or on more rare occasions, later in life. The physiology at presentation relates to the structural morphology and may include cyanosis, low cardiac output (shock), arterial or venous obstruction, excessive pulmonary blood flow, and congestive heart failure. It has long been recognized that infants presenting with symptomatic congenital heart disease have an overall very poor prognosis if left untreated. On the less structurally complex end of the spectrum, patients may present with defects between

This article is a commentary on the following article: $10.1007 /$ s12265011-9289-8.

\footnotetext{
C. D. Fraser Jr. $(\bowtie)$

Department of Congenital Heart Surgery,

Texas Children's Hospital,

Houston, TX, USA

e-mail: CDFraser@texaschildrens.org

C. D. Fraser Jr.

Baylor College of Medicine,

Houston, TX, USA
}

cardiac chambers in isolation or in combination with other problems. Defects of the atrial or ventricular septa are most commonly observed. Again, there is very broad range of symptomatology and physiology relating to the type, size, location, and number of septal defects. For larger defects, with significant intracardiac shunting of blood, the patient's short- and long-term prognosis may be significantly affected if the lesion is left untreated.

Surgical, and more recently, catheter-based treatments for serious congenital cardiac defects represent a true success story of modern medicine. The earliest palliative surgeries, performed in the 1930s (ligation of patent ductus arteriosus and coarctation repair) and 1940s (creation of a systemicto-pulmonary artery shunt) ushered in the era of physiologic treatment for congenital heart disease. In subsequent decades, enormous progress was made in addressing more and more complex conditions including aggressive reconstructions. In the 1950s, cardiopulmonary bypass to support the systemic circulation offered the prospect of intracardiac correction (tetralogy of Fallot, atrioventricular canal, and atrial/ventricular septal defects). In the 1960s and 1970s, durable palliations were developed for single ventricle (Glenn and Fontan procedures). The 1980s saw increasingly aggressive operations for serious defects in newborns and small babies (transposition of the great arteries, truncus arteriosus, aortic interruption, and ultimately, very complex lesions including hypoplastic left heart syndrome). In the 1990s and the first decade of the new millennium, treatments have been further refined to improve operative survival, reduce morbidity, and consider long-term outlook including neurodevelopmental opportunities. Recent striking advances have included successful surgical correction of complex lesions in very small, preterm neonates, the development and implementation of catheter-based treatments for many conditions, and in utero interventions for fetuses with critical cardiac conditions. 
In the setting of this brief overview of the advances that have occurred over the now almost six decades of treatment of congenital heart disease, it must be emphasized that the field still faces an enormous challenge. In fact, each successive generation of "successful" treatment has resulted in the recognition of issues related to the unnatural (or after treatment) history of these various lesions over the course of long-term follow-up. One example is found in the development of an entirely new field of medicine focused on survivors of treatments for complex congenital cardiac lesions who have become adults (adults with congenital heart disease). This subspecialty has arisen, by necessity, for the treatment of ongoing structural and physiologic cardiac derangements in patients that may present years or even decades after the index repair. The "poster child" lesion that offers a great representation of these issues is tetralogy of Fallot. In this condition, several interrelated structural defects combine to create a serious, frequently fatal cyanotic cardiac condition. The basic elements of tetralogy include a large ventricular septal defect and malalignment of the ventricular septum (relative to the aorta) resulting in greater or lesser degrees of right ventricular outflow tract obstruction. The pathophysiology relates to shunting of deoxygenated blood through the ventricular septal defect due to right ventricular outflow tract obstruction. Surgical repair is accomplished by patch closure of the ventricular septal defect (Dacron, expanded PTFE, autologous pericardium) and right ventricular outflow tract resection or incision (often in association with a pulmonary valve incision and right ventricular outflow tract patch). Current expectations for tetralogy repair in major centers include operative survival rates approaching $100 \%$. In the early era of these successful corrections, it was mistakenly concluded that patients were cured of their problem, e. g., having structurally sound hearts with functional capacity for a normal lifespan. The decades of followup have disproven this notion. Patients with repaired tetralogy face lifelong cardiac risk related to their intrinsic structural abnormality and the related imperfections of surgical repair. Ongoing issues include absence of contractility of the septal patch, altered electromechanical properties creating risk of dysrhythmia, calcification of patches and suture material, the deleterious effects of incisions in myocardium with subsequent scar formation, and pulmonary valve insufficiency. Finally, it must be emphasized that the potential liability of these problems is magnified by the fact that primary repairs are performed in infancy and thusly the patient's followup profile is measured in terms of decades, very different from the setting of adults with acquired cardiac conditions undergoing repair.

This historical overview is provided to offer a perspective about the scope of possibilities that exists for bioengineered tissues, blood vessels, valves, and even whole organs to impact the care of patients with congenital cardiac disease. In my estimation, bioengineering offers the potential of a quantum leap forward in treatments. The accompanying article by my colleague Jeffrey Jacot, $\mathrm{PhD}$ and his associate provides insight into current directions being explored for the construction of bioengineered patches and partial cardiac constructs. As a surgeon, my opinion is that the strategic scientific direction within the bioengineering community should be focused on those avenues of investigation that offer the most proximal potential for clinical application. Said differently, it seems logical to start with the least complex problems. As such, creating bioengineered cardiac patches may represent the most rapidly achievable opportunity to bring scientific developments into clinical application. In this regard, the remainder of this commentary will focus on what an "ideal" replacement cardiac patch should offer from the standpoint of a clinician. The opinions offered herein relate to my estimation of the practical issues/opportunities and the challenges relative to current therapies.

To my view, the applications for bioengineered cardiac patches most likely to be achievable include the closure of atrial and ventricular septal defects and functional right ventricular outflow tract patches. Each of these prospects has its own unique structural, electrical, and functional issues, but nonetheless, relative to other needs in congenital cardiac reconstruction, these issues are probably less challenging. Specifically, constructing left ventricular functional patches for large areas of infarcted myocardium or even the more complex notion of using patch material to construct an independently functioning ventricle appears a farfetched goal.

\section{Implantation/Technical Issues}

The ideal reparative patch for closure of atrial septal defects (ASDs), ventricular septal defects (VSDs), and right ventricular outflow tracts should be easy to implant. This is a mandatory attribute given that currently available techniques are straightforward, reproducible, and applicable at very low risk. Implantation of a bioengineered patch must be possible at the same or lower risk. This has been a significant recent issue in the development of catheterbased therapies for VSD closure; the device recently tested in a multicenter clinical trial had an unacceptable complication rate. For surgical implantation to be possible, the patch must be relatively easy to tailor to the individual patient on the operating table. The patch must hold sutures, be malleable, and have limited porosity at the time of implantation (of variable concern when comparing ASD to VSD closure). The structure of the patch must be consistent 
with the necessary abutment with delicate structure including the conduction system (atrioventricular node and bundle of His), valve tissue (tricuspid and aortic), and myocardium.

\section{Mechanical/Structural Properties}

When patches are placed in the setting of immediate exposure to systemic blood pressure (minimum systolic blood pressure of $60-70 \mathrm{mmHg}$ to as high as $>150 \mathrm{mmHg}$ or more), structural integrity is critical. Furthermore, this structural integrity must be durable for the course of a normal lifespan if one considers closing VSDs in newborns. An ideal patch will contribute to the functional contractility of the involved cardiac chamber. This property is especially attractive in the setting of large patches in small children. Of course, a challenging aspect of mechanical contribution to cardiac function relates to appropriate electrical integration and action potential propagation. A consistent finding of currently available patch material is that there is a zone of conduction blockade and the potential of re-entrant circuits and ectopic foci. This potential is exacerbated by the propensity of some patches, especially Dacron and homograft material to scar and calcify. To achieve these properties, an ideal bioengineered patch will have the ability to heal seamlessly with adjacent tissues and achieve a low profile configuration consistent with the adjacent endocardium.

\section{Short-/Long-term Biological Properties}

There are several other very desirable properties for an ideal bioengineered patch. First, the patch should be capable of somatic growth. There are no patches currently capable of this possibility; this translates into the need for some degree of patch redundancy in those cases where a long tunnel connection is created to close a VSD or enlarge the right ventricular outflow tract. Ideally, a bioengineered patch would be genetically identical to the recipient making autologous cell sources most attractive. At minimum, a patch should elicit minimal immunologic and inflammatory reaction.

\section{Practical Considerations}

From a practical standpoint, if the operation or procedure to implant a bioengineered patch is more difficult, complicated, or time consuming, it is unlikely to achieve wide acceptance. This fact will be particularly important in implementing patches for septal defects where there are already good, albeit imperfect, options. Furthermore, a suitable bioengineered option must be readily available; this will require either some option for ongoing storage or advanced preparation. The latter prospect will, by definition, limit patch use to elective or semi-elective circumstances. The cost of the bioengineered patch must be acceptable to current medical practice, again with emphasis on the current available options. Finally, a bioengineered option must pass the scrutiny of regulatory approval. This consideration may be particularly challenging in children where the economics of industry support relative to the comparatively small pediatric market is often an unattractive situation for research and development investment. Furthermore, the regulatory approval pathway for children's devices is arduous and time consuming.

\section{Conclusion}

While the challenges noted previously are significant, the potential for cardiac bioengineering to positively impact patient care is compelling and exciting. Furthermore, it is hoped that progress in the field of constructing effective patches will pave the way for application of more complex cardiac structures including heart valves, vessels, conduction systems, and ultimately, entire organs. 